\title{
On the Kneser-Tits problem for triality forms
}

\author{
Gopal Prasad
}

Dedicated to G. A. Margulis

\begin{abstract}
The purpose of this paper is to provide a concrete description of the "Whitehead group" $W(k, G):=G(k) / G(k)^{+}$for the simply connected triality forms $G$ of $k$-rank 1 , and to use this description to prove that if $k$ is a global field, then the Kneser-Tits problem for these forms has an affirmative solution.
\end{abstract}

Mathematics Subject Classification (2000). $20 \mathrm{G} 30$.

Keywords. Kneser-Tits problem, triality forms.

\section{Introduction}

Let $G$ be a semi-simple simply connected algebraic group defined, simple, and isotropic over a field $k$. Let $G(k)^{+}$be the normal subgroup of $G(k)$ generated by the $k$-rational elements of the unipotent radicals of parabolic $k$-subgroups of $G$. The Kneser-Tits problem is the question whether $G(k)^{+}=G(k)$, or, equivalently, whether $G(k)$ is projectively simple (i.e., the only proper normal subgroups of $G(k)$ are central). In [8] it has been shown that the Kneser-Tits problem has an affirmative solution for all semi-simple simply connected groups defined, simple, and isotropic over $k$, if and only if, the problem has an affirmative solution for every such group of $k$-rank 1 .

It is known that for all $G$ as above, the Kneser-Tits problem has an affirmative solution if $k$ is a local field (see [8]); if $k$ is a global field, then for all $G$ as above of $k$-rank $\geqslant 2$, the Kneser-Tits problem has an affirmative solution. In fact, the only absolutely simple simply connected isotropic groups over global fields for which a solution of the Kneser-Tits problem does not exist in the literature are the triality forms ${ }^{3,6} D_{4,1}^{9}$, and the outer form ${ }^{2} E_{6,1}^{35}$, we have used here the notation for indices introduced in [14], this notation will be used throughout the paper. (Though the groups of type ${ }^{2} E_{6,1}^{29}$ are not included in [15] among the groups for which the Kneser-Tits problem was known to have an affirmative solution, Skip Garibaldi pointed out to me that in [16] Veldkamp has shown that for every absolutely simple simply connected 
group of type ${ }^{2} E_{6,1}^{29}$ over an arbitrary field of characteristic different from 2 and 3 , the Kneser-Tits problem has an affirmative solution. For a modern rendition of Veldkamp's proof, see [4]. We note here that over a global function field, groups of type ${ }^{2} E_{6,1}^{29}$ do not exist since Harder's Galois-cohomology vanishing theorem implies that over these fields the only absolutely simple anisotropic groups are of (inner or outer) type $A_{n}$ for some $n$.)

The purpose of this paper is two fold. First, in $\S 2$, we provide a concrete description of the "Whitehead group" $W(k, G):=G(k) / G(k)^{+}$for the simply connected triality forms $G$ of $k$-rank 1 . Next, in $\S 3$ we use this description to prove that if $k$ is a global field, then for a simply connected triality form $G / k$ of $k$-rank $1, G(k)^{+}=G(k)$ (Theorem 3.1). Theorem 3.1 settles the Kneser-Tits problem for the triality forms over global fields in the affirmative. It was proved jointly with M. S. Raghunathan in the late eighties but that proof has not been published.

We note here that over any field $k$, a triality form of $k$-rank $>1$ is quasi-split (and is actually of $k$-rank 2). It is known (due to C. Chevalley and R. Steinberg, see [15]) that for a semi-simple simply connected quasi-split $k$-group $H, H(k)^{+}=H(k)$.

\section{Description of $G(k) / G(k)^{+}$}

In this section, $k$ is an arbitrary infinite field and $G$ is an absolutely simple simply connected algebraic group of type ${ }^{3,6} D_{4}$ defined over $k$ and of $k$-rank 1.

Let $S$ be a nontrivial $k$-split torus of $G, M=Z_{G}(S)$ its centralizer, and $M^{\prime}=$ $[M, M]$ the commutator subgroup of $M$. Let $P=M \ltimes U$ and $P^{-}=M \ltimes U^{-}$ be the two minimal parabolic $k$-subgroups of $G$ containing $M$. It is known (see, for example, [1, 6.2(v) and 6.11(i)]) that $G(k)^{+}=\left\langle U(k), U^{-}(k)\right\rangle$, i.e., $G(k)^{+}$is generated by $U(k)$ and $U^{-}(k)$, and $G(k)=\left\langle P(k), P^{-}(k)\right\rangle=M(k) G(k)^{+}$. Let $M(k)^{+}=M(k) \cap G(k)^{+}$. Then $W(k, G)=G(k) / G(k)^{+} \cong M(k) / M(k)^{+}$.

From the description of $G$ as the Galois-twist of the corresponding simply connected quasi-split group by a 1-cocycle taking values in a parabolic subgroup corresponding to $P$ in the adjoint group of the quasi-split group, we see that there exists a quaternion division algebra $D$, whose center $K$ is a separable cubic extension of $k$, and whose corestriction ${ }^{1}$ to $k$ is trivial, such that $M$ is the inverse image of the unique 1-dimensional $k$-split torus $\mathrm{GL}_{1, k}$ contained in $R_{K / k}\left(\mathrm{GL}_{1}\right)=\mathrm{GL}_{1, K}$ under the reduced norm map $\mathrm{GL}_{1, D} \stackrel{\mathrm{Nrd}}{\longrightarrow} \mathrm{GL}_{1, K} ; M$ is a connected reductive $k$-group of

\footnotetext{
${ }^{1}$ For a description of the corestriction $\operatorname{Br}(K) \rightarrow \operatorname{Br}(k)$, from the Brauer group $\operatorname{Br}(K)$ of $K$ to the Brauer group $\operatorname{Br}(k)$ of $k$, see [2, §8]. The following is an alternate description of this corestriction. If we identify $\operatorname{Br}(K)$ (resp., $\operatorname{Br}(k))$ with the Galois-cohomology group $H^{2}\left(K, \mathrm{GL}_{1}\right)=H^{2}\left(k, R_{K / k}\left(\mathrm{GL}_{1}\right)\right)$ (resp., $\left.H^{2}\left(k, \mathrm{GL}_{1}\right)\right)$, then the corestriction is the homomorphism $H^{2}\left(k, R_{K / k}\left(\mathrm{GL}_{1}\right)\right) \rightarrow H^{2}\left(k, \mathrm{GL}_{1}\right)$ induced by the norm map $N_{K / k}: R_{K / k}\left(\mathrm{GL}_{1}\right) \rightarrow \mathrm{GL}_{1}$.
} 
dimension 10 and $M^{\prime}=\mathrm{SL}_{1, D}$. From this description of $M$ it is obvious that

$$
M(k)=\left\{x \in D^{\times} \mid \operatorname{Nrd}(x) \in k^{\times}\right\} .
$$

Remark 1. If $k$ is a local field and $K$ is a separable cubic extension of $k$, then the corestriction to $k$ of the unique quaternion division algebra with center $K$ is nontrivial (see [7, Proposition 6.14]). Hence, over a local field $k$, any $k$-isotropic triality form is quasi-split.

In particular, if $v$ is a place of a global field $k, k_{v}$ is the completion of $k$ at $v$, and $K_{v}:=K \otimes_{k} k_{v}$ is a field, then $G$ is quasi-split over $k_{v}$.

Remark 2. A quaternion division algebra $D$ with center $K$ contains an element $x \in D \backslash K$ such that $x^{2} \in K$. (In fact, for any separable quadratic extension $L$ of $K$ contained in $D$, by the Skolem-Noether theorem there is $x \in D^{\times}$such that conjugation by $x$ keeps $L$ stable, and restricted to $L$ it coincides with the nontrivial automorphism of $L / K$. Then $x^{2} \in K$.) This implies that there does not exist a quaternion division algebra whose center is a perfect field of characteristic 2 . Now if $k$ is a perfect field, then so is its separable cubic extension $K$, and we conclude that there does not exist a triality form of rank 1 over a perfect field $k$ of characteristic 2 .

Let

$$
\left.\mathfrak{M}:=\left\langle\ell^{\times}\right| \ell \text { is a separable quadratic extension of } k \text { contained in } D\right\rangle .
$$

Our goal in this section is to prove that $\mathfrak{M}$ is contained in $M(k)^{+}$, and these two groups are equal if, for example, $k$ is not of characteristic 2 . As $\mathfrak{M}$ is clearly a normal subgroup of $D^{\times}$, it would follow, in particular, that if $\operatorname{char}(k) \neq 2$, then $M(k)^{+} \triangleleft D^{\times}$. We begin with the following:

Proposition 2.1. Given a separable quadratic extension $\ell$ of $k$ contained in $D$, there is an absolutely simple simply connected $k$-subgroup $H$ of $G$ of type ${ }^{2} A_{2}$ which contains $S$ and which is quasi-split over $k$, so that $T(k)=\ell^{\times}$, where $T(\subset M)$ is the centralizer of $S$ in $H$.

Proof. Let $T$ be the Zariski-closure of $\ell^{\times}(\subset M(k))$ in $M$. Then $T$ is a 2-dimensional torus containing $S$, and it splits over $\ell$. We now observe that since $D \otimes_{k} \ell$ is a matrix algebra over the field $K \otimes_{k} \ell, M$ is quasi-split over $\ell$ and $T$ is a maximal $\ell$-split torus of $M$. Hence, there exists a Borel subgroup $B$ of $M$ defined over $\ell$ and containing the centralizer $Z$ of $T$ in $M$. Note that $Z$ is a maximal torus of $M$, and hence also of $G$, and it is defined over $k$ since $T$ is. Moreover, $T$ is clearly the maximal $\ell$-split subtorus of $Z$.

We now work with the ordering on the root system of $G$, with respect to the maximal torus $Z$, determined by the Borel subgroup $B \ltimes U$ of $G$. Let $\left\{\alpha_{i}, i=0,1,2,3\right\}$ 
be the set of simple roots enumerated so that for all nonzero $i, \alpha_{i}$ is trivial on $S$. Then $\alpha_{1}, \alpha_{2}$ and $\alpha_{3}$, and their negatives, are the roots of $M$ with respect to $Z$. Let $L$ be the splitting field of $Z$ over $k ; L$ contains $\ell$ since $Z$ contains $T$. Let $\Gamma$ be the Galois group of $L / k$ and $\Gamma^{\prime}$ be the (normal) subgroup of $\Gamma$ of index two consisting of the elements which fix $\ell$ pointwise. $\Gamma^{\prime}$ fixes the root $\alpha_{0}$ and acts transitively on the set $\left\{\alpha_{1}, \alpha_{2}, \alpha_{3}\right\}$. Also, since $U$ is defined over $k$, the set $\Theta$ of positive roots $\alpha=\sum_{i=0}^{3} m_{i} \alpha_{i}$ such that $m_{0}$ is nonzero, which is the set of roots of $U$ with respect to $Z$, is stable under $\Gamma$. The roots $\alpha_{0}, \sum_{i=0}^{3} \alpha_{i}$, and the highest root $2 \alpha_{0}+\sum_{i=1}^{3} \alpha_{i}$ are the only roots in the set $\Theta$ which are fixed under $\Gamma^{\prime}$, and the highest root is actually fixed by all of $\Gamma$. We conclude that the set $\left\{\alpha_{0}, \sum_{i=0}^{3} \alpha_{i}\right\}$ is stable under $\Gamma$. Hence the subgroup $H$ of $G$ generated by the 1-dimensional unipotent root subgroups corresponding to the roots $\alpha_{0}, \sum_{i=0}^{3} \alpha_{i}, 2 \alpha_{0}+\sum_{i=1}^{3} \alpha_{i}$, and their negatives, is a connected subgroup of $G$ defined over $k$, split over $\ell$, and it is clearly of type $A_{2}$. It can be easily checked that $H$ is simply connected. As it contains the subgroup generated by the root subgroups corresponding to the highest root $2 \alpha_{0}+\sum_{i=1}^{3} \alpha_{i}$ and its negative, which is a group $k$-isomorphic to $\mathrm{SL}_{2}$, we conclude that $H$ is of $k$-rank 1 and hence it is a quasi-split special unitary group in three variables defined in terms of the quadratic extension $\ell$. As $T$ is the maximal $\ell$-split subtorus of $Z, T$ is contained in $H$ and it equals the centralizer of $S$ in $H$.

Proposition 2.2. $\mathfrak{M} \subset M(k)^{+}$.

Proof. Let $\ell$ be a separable quadratic extension of $k$ contained in $D$, and $H$ and $T$ be as in the above proposition. Since $H$ is quasi-split over $k$ and is simply connected, $H(k)=H(k)^{+} \subset G(k)^{+}$. This implies that $\ell^{\times}=T(k) \subset M(k)^{+}$, which in turn implies that $\mathfrak{M} \subset M(k)^{+}$.

Notation 2.3. For $u \in U(k) \backslash\{1\}$, let $u=f(u) n(u) g(u)$ be the Bruhat decomposition of $u$ (with respect to $P^{-}$), with $f(u), g(u) \in U^{-}(k)$ and $n(u) \in N(k)$, where $N$ is the normalizer of $S$ in $G$. Note that as $G$ is of $k$-rank $1, N(k) / M(k)$ is of order 2, and $n(u) \in N^{\prime}(k)$, where $N^{\prime}=N \backslash M$.

Let $a$ be the generator of the character group of $S$ which is a root of $U$ with respect to $S$. Then $\pm a, \pm 2 a$ are the roots of $G$ with respect to $S$. Let $U_{b}$ denote the connected unipotent root subgroup corresponding to the root $b \in\{ \pm a, \pm 2 a\}$. It is easy to see that $U_{ \pm a}$ are 9-dimensional, $U_{ \pm 2 a}$ are 1-dimensional, and so $U_{a} / U_{2 a}$ is an 8-dimensional $M$-module defined over $k$; $U=U_{a}, U^{-}=U_{-a}$.

From a consequence of Proposition 1.8 of [3], stated in the first sentence on p. 311 of that paper (note that the notation used in [3] is different from ours), we at once obtain that

$$
M(k)^{+}=\left\langle n(u) n(v)^{-1} \mid u \in U_{a}(k) \backslash U_{2 a}(k), v \in U_{2 a}(k) \backslash\{1\}\right\rangle .
$$


Let $\mathfrak{U}$ denote the set of elements $u \in U_{a}(k) \backslash U_{2 a}(k)$ such that there is an absolutely simple simply connected quasi-split $k$-subgroup $H(u)$ of $G$ of type ${ }^{2} A_{2}$ containing $S, U_{ \pm 2 a}$, and the element $u$ (and hence also $n(u)$ ). We note that if $T$ $(\subset M)$ is the centralizer of $S$ in $H(u)$, and $\ell$ is the separable quadratic extension of $k$ over which $T$ splits, then the $k$-subalgebra of $D$ generated by $T(k)$ is isomorphic to $\ell$ and will be identified with it. With this identification, $T(k)=\ell^{\times}$. Hence, for all $v \in U_{2 a}(k) \backslash\{1\}, n(u) n(v)^{-1} \in T(k)=\ell^{\times} \subset \mathfrak{M}$.

We will now prove the following:

Proposition 2.4. $\mathfrak{M}=\left\langle n(u) n(v)^{-1} \mid u \in \mathfrak{U}, v \in U_{2 a}(k) \backslash\{1\}\right\rangle$.

Proof. To prove the proposition, it would suffice to show that given a separable quadratic extension $\ell$ of $k$ contained in $D$, the group generated by $\left\{n(u) n(v)^{-1}\right.$ | $\left.u \in \mathfrak{U}, \quad v \in U_{2 a}(k) \backslash\{1\}\right\}$ contains $\ell^{\times}$. According to Proposition 2.1, there is an absolutely simple simply connected $k$-subgroup $H$ of $G$ of type ${ }^{2} A_{2}$, which contains $S$ and which is quasi-split over $k$, so that if $T$ is the centralizer of $S$ in $H$, then $T(k)=\ell^{\times}$. This subgroup is $k$-isomorphic to the special unitary group $\mathrm{SU}(h)$ of the hermitian form $h$ on $\ell^{3}$ given by

$$
h(x, y, z)=x \bar{z}+\bar{x} z+y \bar{y} \quad \text { for } x, y, z \in \ell,
$$

where for $\lambda \in \ell, \bar{\lambda}$ denotes its conjugate over $k$. We identify $H$ with $\mathrm{SU}(h)$ in terms of a $k$-isomorphism which carries $T(k)$ onto the diagonal subgroup of $\mathrm{SU}(h)(k)$.

It is obvious that $H(k) \cap\left(U_{a}(k) \backslash U_{2 a}(k)\right) \subset \mathfrak{U}$ (for every $u \in H(k) \cap\left(U_{a}(k) \backslash\right.$ $\left.U_{2 a}(k)\right)$, we can take $H(u)$ appearing in the definition of $\mathfrak{U}$ to be $\left.H\right)$. We easily see, by a direct computation in $\mathrm{SU}(h)(k)$, for any field $k$, that the elements $n(u) n(v)^{-1}$, as $u$ varies over $H(k) \cap\left(U_{a}(k) \backslash U_{2 a}(k)\right)$ and $v$ varies over $H(k) \cap\left(U_{2 a}(k) \backslash\{1\}\right)$, generate $T(k)=\ell^{\times}$. This proves the proposition.

It follows from Theorem 5.1 of Raghunathan [9] that given an element $u \in$ $U_{a}(k) \backslash U_{2 a}(k), u^{2} \neq 1$, as $U_{2 a}$ is 1 -dimensional, there is an absolutely simple simply connected quasi-split $k$-subgroup $H=H(u)$, of type ${ }^{2} A_{2}$, containing the subgroups $S, U_{ \pm 2 a}$, and the element $u$. Thus $\mathfrak{U} \supset\left\{u \in U_{a}(k) \backslash U_{2 a}(k) \mid u^{2} \neq 1\right\}$. In particular, if $\operatorname{char}(k) \neq 2$, then as every nontrivial element of $U_{a}(k)$ is of order at least $3, \mathfrak{U}=U_{a}(k) \backslash U_{2 a}(k)$. Now combining this with the description of $\mathfrak{M}$ given by Proposition 2.4 and that of $M(k)^{+}$provided by $(*)$, we conclude the following.

Theorem 2.5. If the characteristic of $k$ is different from 2 , then $M(k)^{+}=\mathfrak{M}$. Hence, $W(k, G) \cong M(k) / \mathfrak{M}$.

The following remark is due to Andrei S. Rapinchuk. 
Remark 3. If the Whitehead group $W(k, G)\left(\cong M(k) / M(k)^{+}\right)$is finite and $M(k)^{+}=$ $\mathfrak{M}$, then $W(k, G)$ is solvable. In fact, $M(k)$ and $M(k)^{+}(=\mathfrak{M})$ are normal subgroups of $D^{\times}$, and the conjugation action of $D^{\times}$on $M(k)$ and $M(k)^{+}$induces an action of $D^{\times}$on $M(k) / M(k)^{+}$. Let $C$ be the kernel of this action. Then $C$ is subgroup of $D^{\times}$ of finite index, and it clearly contains $M(k)^{+}$. Hence, according to the main theorem of [11], the group $D^{\times} / C$ is solvable. This implies, in particular, that $M(k) / C \cap M(k)$ is solvable. On the other hand, the commutator subgroup $[M(k), C]$ is contained in $M(k)^{+}$, and hence, $(C \cap M(k)) / M(k)^{+}$is abelian, and we conclude that $M(k) / M(k)^{+}$ is solvable.

For the proof of Theorem 3.1, the following proposition (Proposition 2.7), which is an immediate consequence of Raghunathan's result mentioned above, suffices. For the sake of completeness, we give its proof which was obtained with help from Rapinchuk.

Let $k$ be an arbitrary field. The central $k$-split torus $S$ of $M$ acts on the 8-dimensional $M$-module $V:=U_{a} / U_{2 a}$ by homotheties by the nontrivial character $a$. Over the algebraic closure $\bar{k}$ of $k, M^{\prime}=[M, M]$ is the direct product of three copies of $\mathrm{SL}_{2}$, $M^{\prime}=M_{1}^{\prime} \times M_{2}^{\prime} \times M_{3}^{\prime}$, where $M_{i}^{\prime} \cong \mathrm{SL}_{2}$ for $i \leqslant 3$. Over $\bar{k}$, as an $M^{\prime}$-module, $V$ is the tensor product $V_{1} \otimes V_{2} \otimes V_{3}$, where $V_{i}$ is the standard 2-dimensional representation of $M_{i}^{\prime}\left(\cong \mathrm{SL}_{2}\right)$, and $S$ acts faithfully on $V$ by homotheties.

We fix a basis $\left\{e_{1}^{(i)}, e_{2}^{(i)}\right\}$ of $V_{i}$, and let $T_{i}$ be the maximal torus of $M_{i}^{\prime}$ represented by diagonal matrices with respect to this basis; we will denote $\operatorname{diag}\left(t, t^{-1}\right)$ by $\delta(t)$. Set

$$
e=e_{1}^{(1)} \otimes e_{1}^{(2)} \otimes e_{1}^{(3)}+e_{2}^{(1)} \otimes e_{2}^{(2)} \otimes e_{2}^{(3)} .
$$

Lemma 2.6. The identity component $M_{e}^{\circ}$ of the isotropy subgroup $M_{e}$ of $e$ in $M$ coincides with

$$
T:=\left\{\left(\delta\left(t_{1}\right), \delta\left(t_{2}\right), \delta\left(t_{3}\right)\right) \in T_{1} \times T_{2} \times T_{3} \mid t_{1} t_{2} t_{3}=1\right\},
$$

and the annihilator of $e$ in the Lie algebra of $M$ is precisely the Lie algebra of the two dimensional torus $T$. Consequently, the $M$-orbit $\Omega:=M \cdot$ e of $e$ is a Zariskiopen subvariety of $V=U_{a} / U_{2 a}$, and for any $k$-rational element $v$ of $\Omega$, the identity component $M_{v}^{\circ}$ of the isotropy subgroup $M_{v}$ is a 2-dimensional torus defined over $k$ and contained in $M^{\prime}$.

Proof. It is easy to see that to prove the first assertion, it is enough to show that

$$
M_{e}^{\prime} \subset N_{1} \times N_{2} \times N_{3},
$$

where $N_{i}$ is the normalizer of $T_{i}$ in $M_{i}^{\prime}$. Assume that $(\diamond)$ does not hold. Then there exists an $i \in\{1,2,3\}$ such that $\pi_{i}\left(M_{e}^{\prime}\right) \not \subset N_{i}$, where $\pi_{i}: M^{\prime} \rightarrow M_{i}^{\prime}$ is the natural 
projection. Since $\pi_{i}\left(M_{e}^{\prime}\right) \supset \pi_{i}(T)=T_{i}$, we see that $\pi_{i}\left(M_{e}^{\prime}\right)$ contains either an upper or lower triangular element. Then, by considering commutators of elements of $M_{e}^{\prime}$ with appropriate elements of $T$, we see that $M_{e}^{\prime}$ contains the subgroup that has the full upper or lower unitriangular group as the $i$-th and the identity as the remaining two components. But it is obvious that such a subgroup cannot fix $e$, and so we have proved the first assertion.

Since $\operatorname{dim} M \cdot e=10-2=8=\operatorname{dim} V$, the orbit $M \cdot e$ is open in $V$.

The annihilator of $e$ in the Lie algebra of $M$ can be easily computed and shown to be equal to the Lie algebra of $T$. The assertion that for a $k$-rational element $v$ of $\Omega=M \cdot e, M_{v}^{\circ}$ is defined over $k$ follows now from the fact that the Lie algebra of $M_{v}$ coincides with the annihilator of $v$ in the Lie algebra of $M$.

Since the $M$-orbit $\Omega=M \cdot e$, being an open subvariety of the $k$-vector space $V$, contains a $k$-rational element, we conclude that $\Omega$ is in fact defined over $k$. Let $U$ be the open $k$-subvariety of $U_{a}$ consisting of the elements whose image in $V$ lies in the open subvariety $\Omega$. Now let $u \in U(k)$ and $v$ be its image in $V$. Then $T_{v}:=M_{v}^{\circ}$ is a torus of dimension 2 which is defined and anisotropic over $k$. The derived subgroup $H(u)$ of the centralizer of $T_{v}$ in $G$ is an absolutely simple simply connected quasisplit $k$-group of type ${ }^{2} A_{2}$ which contains $S, U_{ \pm 2 a}$, and the element $u$. Thus we have proved the following.

Proposition 2.7. Let $U$ be the nontrivial open $k$-subvariety of $U_{a}$ defined above. Then $U(k) \subset \mathfrak{U}$.

\section{The Kneser-Tits problem for triality forms over global fields}

The purpose of this section is to prove the following result.

Theorem 3.1 (Prasad and Raghunathan). Let $k$ be a global field (i.e., either a number field, or the function field of a curve over finite field) and $G$ be an absolutely simple simply connected algebraic $k$-group of type ${ }^{3,6} D_{4}$ of $k$-rank 1 . Then $G(k)^{+}=G(k)$.

We will continue to use the notation introduced in $\$ 2$, however, henceforth $k$ will denote a global field. For a global field $L$, and a place $w$ of $L$, we will denote by $L_{w}$ the completion of $L$ at $w$. For a place $v$ of $k$, we will denote $D \otimes_{k} k_{v}$ by $D_{v}$ in the sequel.

As observed at the beginning of $\S 2$, we may identify $M(k)$ with $\left\{x \in D^{\times}\right.$। $\left.\operatorname{Nrd}(x) \in k^{\times}\right\}$. In particular, $\mathrm{SL}_{1}(D) \subset M(k) \subset G(k)$. To prove Theorem 3.1, we must show that $M(k)^{+}=M(k)$. Our first goal is to show that $\mathrm{SL}_{1}(D) \subset M(k)^{+}$. 
3.2. Let $\mathcal{T}$ be the finite set of places of $k$ where $G$ is not quasi-split. Let $v \in \mathcal{T}$. Then $K \otimes_{k} k_{v}$ cannot be a field, for if it is a field, then $G$ is a triality form over $k_{v}$, and according to Remark 1, it is quasi-split at $v$. Hence, there are only the following two possibilities.

(1) There are three distinct places $w_{1}, w_{2}$ and $w_{3}$ of $K$ lying over $v$, i.e., $K \otimes_{k} k_{v}=$ $K_{w_{1}} \oplus K_{w_{2}} \oplus K_{w_{3}}$, and each $K_{w_{i}}$ is equal to $k_{v}$. As the corestriction of $D_{v}$ to $k_{v}$ is trivial, $D_{v}=D \otimes_{k} k_{v}=D \otimes_{K}\left(K \otimes_{k} k_{v}\right)=M_{2}\left(k_{v}\right) \oplus \mathscr{D} \oplus \mathscr{D}$, where $\mathscr{D}$ is the quaternion division algebra with center $k_{v}$. In this case, over $k_{v}, G$ is an inner-form of rank 2 of the simply connected Chevalley group of type $D_{4}$.

From the description of $M$ as an algebraic $k$-group given in $\S 2$, it is obvious that an element $(x, y, z) \in D_{v}^{\times}=\mathrm{GL}_{2}\left(k_{v}\right) \times \mathscr{D}^{\times} \times \mathscr{D}^{\times}$is in $M\left(k_{v}\right)$ if and only if $\operatorname{det}(x)=\operatorname{Nrd}(y)=\operatorname{Nrd}(z)$.

(2) There are exactly two places $w_{1}, w_{2}$ of $K$ lying over $v$, i.e., $K \otimes_{k} k_{v}=$ $K_{w_{1}} \oplus K_{w_{2}}$, we can assume that $K_{w_{1}}=k_{v}$, and $K_{w_{2}}$ is a separable quadratic extension of $k_{v}$. In this case, $K$ is not a Galois extension of $k, G$ is of type ${ }^{6} D_{4}$ over $k$ and of type ${ }^{2} D_{4}$ over $k_{v}$. As the corestriction of $D_{v}$ to $k_{v}$ is trivial, $D_{v}=D \otimes_{k} k_{v}=$ $D \otimes_{K}\left(K \otimes_{k} k_{v}\right)=\mathscr{D}_{1} \oplus \mathscr{D}_{2}$, where $\mathscr{D}_{i}$ is a quaternion division algebra with center $K_{w_{i}}, i=1,2$. In particular, $v$ is necessarily nonarchimedean in this case.

From the description of $M$ as an algebraic $k$-group given in $\S 2$, we see that $M\left(k_{v}\right)$ is the set of elements $(x, y) \in D_{v}^{\times}=\mathscr{D}_{1}^{\times} \times \mathscr{D}_{2}^{\times}$such that $\operatorname{Nrd}(x)=\operatorname{Nrd}(y)$.

Lemma 3.3. For every place $v \in \mathcal{T}$, we have $\left[D_{v}^{\times}, M\left(k_{v}\right)\right]=\mathrm{SL}_{1}\left(D_{v}\right)$.

Proof. The lemma can be proved easily using the description of $D_{v}^{\times}$and $M\left(k_{v}\right)$ given above and the following well-known results: (i) $\left[\mathrm{SL}_{2}\left(k_{v}\right), \mathrm{SL}_{2}\left(k_{v}\right)\right]=\mathrm{SL}_{2}\left(k_{v}\right)$. For any central division algebra $\mathfrak{D}$ over a local field $\ell$, (ii) $\left[\mathfrak{D}^{\times}, \mathfrak{D}^{\times}\right]=\mathrm{SL}_{1}(\mathfrak{D})$ (this is a theorem of Matsushima and Nakayama), (iii) $\operatorname{Nrd}\left(\mathfrak{D}^{\times}\right)=\ell^{\times}$if $\ell$ is nonarchimedean, (iv) $\left[\mathrm{SL}_{1}(\mathfrak{D}), \mathrm{SL}_{1}(\mathfrak{D})\right]$ is the first congruence subgroup of $\mathrm{SL}_{1}(\mathfrak{D})$ (see the corollary in $\S 5$ of [13]). (v) If case (2) of 3.2 occurs and $K_{w_{2}}$ is an unramified extension of $k_{v}$, then in the quaternion division algebra $\mathcal{D}_{2}$ we can find a uniformizer which normalizes an unramified quadratic extension of $K_{w_{2}}$ contained in $\mathscr{D}_{2}$ and whose square is an element of $k_{v}$.

Let $G_{\mathcal{T}}=\prod_{v \in \mathcal{T}} G\left(k_{v}\right)$. We will think of $G(k)$ as a subgroup of $G_{\mathcal{T}}$ in terms of its diagonal embedding in the latter. The $\mathcal{T}$-adic topology on a subset of $G(k)$ is the topology induced on it by the product (locally compact Hausdorff) topology on $G_{\mathcal{T}}$.

Now we observe that the map $U(k) \backslash\{1\} \rightarrow N^{\prime}(k)$, which maps $u$ onto $n(u)$ (see Notation 2.3), is continuous in the $\mathcal{T}$-adic topology. To see this, we note that $\Omega^{\prime}:=$ $U^{-} N^{\prime} U^{-}$is an open $k$-subvariety of $G, \Omega^{\prime}(k)$ contains $U(k) \backslash\{1\}$, and the product morphism $U^{-} \times N^{\prime} \times U^{-} \rightarrow G$ is an isomorphism onto $\Omega^{\prime}$. The inverse of the product morphism induces a homeomorphism from $\Omega_{\mathcal{T}}^{\prime}:=\prod_{v \in \mathcal{T}} U^{-}\left(k_{v}\right) N^{\prime}\left(k_{v}\right) U^{-}\left(k_{v}\right)$ 
onto $\prod_{v \in \mathcal{T}} U^{-}\left(k_{v}\right) \times \prod_{v \in \mathcal{T}} N^{\prime}\left(k_{v}\right) \times \prod_{v \in \mathcal{T}} U^{-}\left(k_{v}\right)$. Let $v$ be the composite of this homeomorphism and the projection onto the middle factor. Then the map $u \mapsto n(u)$ is simply the restriction of $v$ to $U(k) \backslash\{1\}$, and hence it is continuous in the $\mathcal{T}$-adic topology.

Lemma 3.4. (1) $G(k)^{+}$is dense in $G_{\mathcal{T}}=\prod_{v \in \mathcal{T}} G\left(k_{v}\right)$.

(2) $M(k)^{+}$and $\mathfrak{M}$ are dense in $M_{\mathcal{T}}:=\prod_{v \in \mathcal{T}} M\left(k_{v}\right)$.

Proof. (1): It is known that $G$ has the weak approximation property (Theorem 7.8 of [7]) and hence $G(k)$ embedded diagonally in $G_{\mathcal{T}}$ is dense in the latter. As $G$ is $k$-isotropic, $G\left(k_{v}\right)$ does not contain any proper noncentral normal subgroups (Theorems 7.1 and 7.6 of [7]). This implies that the noncentral normal subgroup $G(k)^{+}$of $G(k)$ is also dense in $G_{\mathcal{T}}$.

(2): Since $G(k)^{+}$is dense in $\prod_{v \in \mathcal{T}} G\left(k_{v}\right)$, it follows that $G(k)^{+} \cap \Omega_{\mathcal{T}}=$ $U(k)\left(M(k) \cap G(k)^{+}\right) U^{-}(k)$ is dense in the open Bruhat-cell

$$
\Omega_{\mathcal{T}}:=\prod_{v \in \mathcal{T}} U\left(k_{v}\right) M\left(k_{v}\right) U^{-}\left(k_{v}\right) .
$$

Projecting on the middle component we conclude that $M(k)^{+}=M(k) \cap G(k)^{+}$is dense in $M_{\mathcal{T}}=\prod_{v \in \mathcal{T}} M\left(k_{v}\right)$.

Now to prove that $\mathfrak{M}$ is dense in $M_{\mathcal{T}}$, it suffices to show that it is dense in $M(k)^{+}$in the $\mathcal{T}$-adic topology. Let $\mathcal{U}$ be the nontrivial open $k$-subvariety of $U_{a}$ as in Proposition 2.7. Then according to that proposition $U(k) \subset \mathfrak{U}$, which implies that the subset $\mathfrak{U}$ of $U_{a}(k) \backslash U_{2 a}(k)$ is dense in the latter in the $\mathcal{T}$-adic topology. Now the density of $\mathfrak{M}$ in $M(k)^{+}$is a consequence of the description of $\mathfrak{M}$ provided by Proposition 2.4, the description of $M(k)^{+}$given by $(*)$, and the continuity of the map $u \mapsto n(u)$ established above.

Proposition 3.5. $\mathrm{SL}_{1}(D) \subset \mathfrak{M}$.

Proof. Consider the subgroup $\mathscr{H}=\left[D^{\times}, \mathfrak{M}\right]$ of $\mathfrak{M}$. Since $D^{\times}$is dense in $\prod_{v \in \mathcal{T}} D_{v}^{\times}$ and $\mathfrak{M}$ is dense in $\prod_{v \in \mathcal{T}} M\left(k_{v}\right)$ (Lemma 3.4(2)), $\mathscr{H}$ is dense in $\prod_{v \in \mathcal{T}}\left[D_{v}^{\times}, M\left(k_{v}\right)\right]$ $=\prod_{v \in \mathcal{T}} \mathrm{SL}_{1}\left(D_{v}\right)$ (where the last equality is given by Lemma 3.3). Therefore, $\mathscr{H}$ is dense in $\operatorname{SL}_{1}(D)$ in the $\mathcal{T}$-topology. However, $\mathcal{H}$ is a noncentral normal subgroup of $\mathrm{SL}_{1}(D)$, so it is $\mathcal{T}$-adically open (and hence closed), see [5], and for $k$ of characteristic 2, see [10]; note that any place $w$ of $K$ such that $D \otimes_{K} K_{w}$ is a division algebra lies over a place $v$ of $k$ contained in $\mathcal{T}$. This implies that $\mathscr{H}=\mathrm{SL}_{1}(D)$.

Let now

$$
\mathcal{A}=\operatorname{Nrd}_{D / K}(M(k)) \subset k^{\times}, \quad \mathscr{B}=\operatorname{Nrd}_{D / K}(\mathfrak{M}) .
$$

Since $\operatorname{SL}_{1}(D) \subset \mathfrak{M} \subset M(k)^{+}$, to prove Theorem 3.1, it is enough to show that $\mathscr{B}=\mathcal{A}$ (this will also imply that $\mathfrak{M}=M(k)$ ). 
Lemma 3.6. An element $a \in k^{\times}$lies in $\mathcal{A}$ if and only if $a$ is positive in $k_{v}=\mathbb{R}$ for all real places $v \in \mathcal{T}$.

Proof. This follows from the norm theorem of Hasse, Schilling and Maass (see Theorem 33.15 of [12]) which asserts that for a finite dimensional division algebra $A$ with center a global field $K$, an element $x \in K^{\times}$is the reduced norm of an element of $A^{\times}$if and only if $x$ is positive at all real places $w$ of $K$ such that $A \otimes_{K} K_{w}$ is a division algebra.

Lemma 3.7. Suppose $\ell$ is a separable quadratic extension of $k$ such that for all $v \in \mathcal{T}$ for which case (1) of 3.2 occurs, $\ell \otimes_{k} k_{v}$ is a field, and for $v \in \mathcal{T}$ for which case (2) of 3.2 occurs, $\ell \otimes_{k} k_{v}$ is a field which is not isomorphic to $K_{w_{2}}$ over $k_{v}$. Then the composite $K \cdot \ell$, and so also $\ell$, is embeddable in $D$.

Proof. Recall that a quadratic extension $L$ of $K$ is embeddable in $D$ if and only if for all places $w$ of $K$ such that $D \otimes_{K} K_{w}$ is a division algebra, $L \otimes_{K} K_{w}$ is a field (see [12]).

Let $L:=K \cdot \ell$ and let $w$ be a place of $K$ such that $D \otimes_{K} K_{w}$ is a division algebra. Then $w$ lies over a place of $k$ belonging to $\mathcal{T}$. Now we observe that $L \otimes_{K} K_{w}=\ell \otimes_{k} K \otimes_{K} K_{w}=\ell \otimes_{k} K_{w}$. Thus it would suffice to show that $\ell \otimes_{k} K_{w}$ is a field for any place $w$ of $K$ lying over a place $v \in \mathcal{T}$.

Given a place $v \in \mathcal{T}$ for which case (1) of 3.2 occurs, for the places $w_{i}, i=1,2,3$, of $K$ lying over $v, K_{w_{i}}$ is isomorphic to $k_{v}$, so $\ell \otimes_{k} K_{w_{i}}$ is a field by hypothesis. On the other hand, if $v$ is a place of $k$ for which case (2) of 3.2 occurs, then $w_{1}, w_{2}$ mentioned there are the places of $K$ lying over $v, K_{w_{1}}$ is isomorphic to $k_{v}$, and hence again, by hypothesis, $\ell \otimes_{k} K_{w_{1}}$ is a field. Finally, as $\ell \otimes_{k} k_{v}$ is a field which is not isomorphic to $K_{w_{2}}, \ell \otimes_{k} K_{w_{2}}$ is a field as well.

We will also need the following well known result of local class field theory.

Lemma 3.8. Let $\mathcal{K}$ be a local field. Then

(1) The correspondence $\mathcal{L} \mapsto N_{\mathscr{L} / \mathcal{K}}\left(\mathcal{L}^{\times}\right)$is a bijection between the set of separable quadratic extensions $\mathcal{L}$ of $\mathcal{K}$ and the set of open subgroups $\mathcal{N}$ of $\mathcal{K}^{\times}$of index 2.

(2) $\mathcal{K}^{\times} /\left(\mathcal{K}^{\times}\right)^{2}$ is a direct sum of at least two copies of $\mathbb{Z} / 2 \mathbb{Z}$, furthermore, if the characteristic of the residue field of $\mathcal{K}$ is 2 , then it is a direct sum of at least three copies of $\mathbb{Z} / 2 \mathbb{Z}$. $\left(\mathcal{K}^{\times} /\left(\mathcal{K}^{\times}\right)^{2}\right.$ is infinite if and only if $\mathcal{K}$ is of characteristic 2.)

Let $\mathcal{T}_{\infty}$ be the set of archimedean places in $\mathcal{T}$, and let $\mathcal{T}_{0}$ be the set of nonarchimedean places $v \in \mathcal{T}$ for which 3.2 (2) holds and the characteristic of the residue field of $k_{v}$ is odd. To show that an element of $\mathcal{A}$ belongs to $\mathcal{B}$ we will use: 
Lemma 3.9. Let $a \in \mathcal{A}$ and assume that for each $v \in \mathcal{T}_{0}$, there exists a separable quadratic extension $\mathcal{L}(v)$ of $k_{v}$ such that $\mathcal{L}(v)$ is not isomorphic (over $k_{v}$ ) to the field $K_{w_{2}}$ of $3.2(2)$, and such that a is the norm of an element of $\mathcal{L}(v)$. Then there exists $b \in k$ such that the polynomial $x^{2}-b x+a$ is irreducible, separable and its splitting field $\ell$ is embeddable in $D$.

Proof. For any real place $v \in \mathcal{T}$, let $b_{v}=0$, so that by Lemma 3.6, $x^{2}-b_{v} x+a$ is irreducible over $k_{v}=\mathbb{R}$, and its splitting field, which we denote by $\mathcal{L}(v)$, is equal to $\mathbb{C}$.

For a nonarchimedean place $v \in \mathcal{T} \backslash \mathcal{T}_{0}$, let $\mathcal{L}(v)$ be a separable quadratic extension of $k_{v}$ such that $a$ is the norm of an element of $\mathcal{L}(v)$, and moreover if 3.2 (2) holds, then $\mathcal{L}(v)$ is not isomorphic to the quadratic extension $K_{w_{2}}$ over $k_{v}$. Note that if $v$ is a nonarchimedean place lying in $\mathcal{T} \backslash \mathcal{T}_{0}$ such that 3.2(2) holds, then the characteristic of the residue field of $k_{v}$ is 2, and as according to Lemma 3.8 (2), $k_{v}^{\times} /\left(k_{v}^{\times}\right)^{2}$ is a direct sum of at least three copies of $\mathbb{Z} / 2 \mathbb{Z}$, there exists a separable quadratic extension $\mathcal{L}(v)$ of $k_{v}$ which is not isomorphic to $K_{w_{2}}$ over $k_{v}$ and such that $a$ is a norm of an element of $\mathcal{L}(v)$.

For $v \in \mathcal{T}_{0}$, let $\mathcal{L}(v)$ be as in the statement of the lemma. For $v \in \mathcal{T}$, nonarchimedean, let $b_{v}$ be the trace of an element of $\mathcal{L}(v) \backslash k_{v}$ whose norm is $a$ (not all elements of norm 1 in $\mathcal{L}(v)^{\times}$are contained in $k_{v}$, so there is an element in $\mathcal{L}(v) \backslash k_{v}$ whose norm is $a$ ). The splitting field of $x^{2}-b_{v} x+a$ over $k_{v}$ is $\mathcal{L}(v)$.

Choose $b \in k$ which approximates $b_{v}$ so closely, for all $v \in \mathcal{T}$, that by Krasner's Lemma the splitting field of $x^{2}-b x+a$ over $k_{v}$ is isomorphic to $\mathcal{L}(v)$.

Let $\ell$ be the splitting field of $x^{2}-b x+a$ over $k$. Then the above choice of $b$ guarantees that $\ell$ satisfies the hypothesis of Lemma 3.7 and hence it is embeddable in $D$.

Now to prove that $\mathscr{B}=\mathcal{A}$, we will use Lemma 3.9. This lemma implies that under certain circumstances, an element $a \in \mathcal{A}$ is actually the norm of an element of a separable quadratic extension $\ell$ of $k$ contained in $D$, and hence it belongs to $\mathscr{B}$. We would like to show that such elements generate $\mathcal{A}$. For this purpose, we need the following lemma.

For $v \in \mathcal{T}_{0}$, let $K_{w_{2}}$ be the field as in 3.2(2), and let $\mathcal{N}_{v} \subset k_{v}^{\times}$be the subgroup of norms of elements of $K_{w_{2}}^{\times}$.

Lemma 3.10. Consider the following condition on an element $x \in k^{\times}$.

$$
\text { For } v \in \mathcal{T}_{0} \text {, either } x \notin \mathcal{N}_{v} \text { or } x \in\left(k_{v}^{\times}\right)^{2} .
$$

Then for each $a \in \mathcal{A}$, there are elements $a_{1}, a_{2} \in \mathcal{A}$, satisfying condition $(\diamond)$, such that $a=a_{1} a_{2}$. 
Proof. Let $a \in \mathcal{A}$. Then for $v \in \mathcal{T}_{\infty}, a$ is positive in $k_{v}=\mathbb{R}$. Observe now that for $v \in \mathcal{T}_{0}, \mathcal{N}_{v}$ is an open (and hence closed) subgroup of index two in $k_{v}^{\times}$. So it contains the subgroup $\left(k_{v}^{\times}\right)^{2}$ and $k_{v}^{\times} \backslash \mathcal{N}_{v}$ is open; moreover, $\left(k_{v}^{\times}\right)^{2}$ is an open subgroup of $k_{v}^{\times}$since (for all $v \in \mathcal{T}_{0}$ ) the characteristic of the residue field of $k_{v}$ is odd.

Consider the open subset

$$
\mathcal{O}:=\prod_{v \in \mathcal{T}_{\infty}} k_{v}^{>0} \prod_{\left\{v \in \mathcal{T}_{0} \mid a \in \mathcal{N}_{v}\right\}}\left(k_{v}^{\times} \backslash \mathcal{N}_{v}\right) \prod_{\left\{v \in \mathcal{T}_{0} \mid a \notin \mathcal{N}_{v}\right\}}\left(k_{v}^{\times}\right)^{2},
$$

of $\prod_{v \in \mathcal{T}_{0} \cup \mathcal{T}_{\infty}} k_{v}^{\times}$. Since $k^{\times}$is dense in the latter group, it follows that $\mathcal{O}$ contains an element $a_{1} \in k^{\times}$. Let $a_{2}=a / a_{1}$. Then it is easy to check that both $a_{1}$ and $a_{2}$ satisfy condition $(\diamond)$. Also, for all $v \in \mathcal{T}_{\infty}, a_{i}$ is positive in $k_{v}=\mathbb{R}$, for $i=1,2$. According to Lemma 3.6, both $a_{1}$ and $a_{2}$ lie in $\mathcal{A}$ and we are done.

We can finally prove

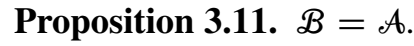

Proof. Let $a \in \mathcal{A}$. In view of Lemma 3.10, we may assume that $a$ satisfies $(\diamond)$. We claim that $a$ is the norm of an element in a separable quadratic extension $\ell$ of $k$ embeddable in $D$. To prove this claim, it suffices to show that $a$ satisfies the hypothesis of Lemma 3.9. By condition $(\diamond)$, for $v \in \mathcal{T}_{0}$, either $a \notin \mathcal{N}_{v}$ or $a \in\left(k_{v}^{\times}\right)^{2}$, and using Lemma 3.8, we can find an open subgroup $\mathcal{M}_{v}$ of $k_{v}^{\times}$of index 2 which contains $a$ but is different from $\mathcal{N}_{v}$. Then the quadratic Galois extension $\mathcal{L}(v)$ of $k_{v}$ determined by $\mathcal{M}_{v}$ is not isomorphic to $K_{w_{2}}$ and $a$ is the norm of an element in $\mathcal{L}(v)$.

Proposition 3.11 completes the proof of Theorem 3.1.

Acknowledgements. It is a pleasure to thank Skip Garibaldi, M. S. Raghunathan and Andrei S. Rapinchuk for helpful conversations and correspondence. I would also like to thank Vladimir Chernousov, Yoav Segev and Jean-Pierre Serre for their interest in this work and for their comments on an earlier draft of this paper.

Support from the Institute for Advanced Study (Princeton), the National Science Foundation (grant no. DMS-0400640), and the BSF (grant no. 200071) is gratefully acknowledged.

Note added in proof. Philippe Gille has recently proved that for an absolutely simple simply connected isotropic triality form $G$ over an arbitrary field $k$, the Whitehead group $W(k, G)$ is trivial, i.e., $G(k)^{+}=G(k)$. He has also settled the Kneser-Tits problem for the last remaining case over global fields of absolutely simple simply connected groups with Tits-index ${ }^{2} E_{6,1}^{35}$ in the affirmative. See the write-up of his Séminaire Bourbaki talk "Le problème de Kneser-Tits" delivered in November 2007. 


\section{References}

[1] A. Borel, J. Tits, Homomorphismes "abstraits" de groupes algébriques simples. Ann. of Math. 97 (1973), 499-571. Zbl 0272.14013 MR 0316587

[2] P. K. Draxl, Skew fields. London Math. Soc. Lecture Note Ser. 81, Cambridge University Press, Cambridge 1983. Zbl 0498.16015 MR 0696937

[3] V. Deodhar, On central extensions of rational points of algebraic groups. Amer. J. Math. 100 (1978), 303-386. Zbl 0392.20027 MR 0489962

[4] S. Garibaldi, Kneser-Tits for a rank 1 form of $E_{6}$ (after Veldkamp). Compositio Math. 143 (2007), 191-200. Zbl 05145745 MR 2295201

[5] G. A. Margulis, On the multiplicative group of a quaternion algebra over a global field. Soviet Math. Dokl. 21 (1980), 780-784. Zbl 0503.12011 MR 0577836

[6] V. P. Platonov, A. S. Rapinchuk, On the group of rational points of three-dimensional groups. Soviet Math. Dokl. 20 (1979), 693-697. Zbl 0431.12012 MR 0545350

[7] V. P. Platonov, A. S. Rapinchuk, Algebraic groups and number theory. Pure Appl. Math. 139, Academic Press, Inc., Boston, MA, 1994. Zbl 0841.20046 MR 1278263

[8] G. Prasad, M. S. Raghunathan, On the Kneser-Tits problem. Comment. Math. Helv. 60 (1985), 107-121. Zbl 0574.20033 MR 0787664

[9] M. S. Raghunathan, On the congruence subgroup problem II. Invent. Math. 85 (1986), 73-117. Zbl 0603.20044 MR 0842049

[10] M. S. Raghunathan, On the group of norm 1 elements in a division algebra. Math. Ann. 279 (1988), 457-484. Zbl 0639.20026 MR 0922429

[11] A. S. Rapinchuk, Y. Segev, G. M. Seitz, Finite quotients of the multiplicative group of a finite dimensional division algebra are solvable. J. Amer. Math. Soc. 15 (2002), 929-978. Zbl 1008.16018 MR 1915823

[12] I. Reiner, Maximal orders. Academic Press, Inc., New York, NY, 1975. Zbl 1024.16008 MR 0393100

[13] C. Riehm, The norm 1 group of a p-adic division algebra. Amer. J. Math. 92 (1970), 499-523. Zbl 0199.37601 MR 0262250

[14] J. Tits, Classification of algebraic semi-simple groups. In Algebraic Groups and Discontinuous Subgroups (Proc. Sympos. Pure Math., Boulder, Colo., 1965), Amer. Math. Soc., Providence, R.I., 1966, 33-62. Zbl 0238.20052 MR 0224710

[15] J. Tits, Groupes de Whitehead de groupes algébriques simples sur un corps (d'après V. P. Platonov et al.). In Séminaire Bourbaki, 29e année (1976/7), Exp. No. 505, 218-236. Zbl 0389.16007 MR 0521771

[16] F. D. Veldkamp, Unitary groups in projective octave planes. Compositio Math. 19 (1968), 213-258. Zbl 0195.50402 MR 0236815

Received January 15, 2007

Gopal Prasad, Department of Mathematics, University of Michigan, Ann Arbor, MI 48109,

U.S.A.

E-mail: gprasad@umich.edu 